\title{
Long-term results and recurrence patterns from SCOPE-1: a phase II/III randomised trial of definitive chemoradiotherapy $+/-$ cetuximab in oesophageal cancer
}

T Crosby ${ }^{1}$, C N Hurt ${ }^{\star}, 2$, S Falk ${ }^{3}$, S Gollins ${ }^{4}$, J Staffurth ${ }^{1}$, R Ray ${ }^{2}, \mathrm{~J} \mathrm{~A} \mathrm{Bridgewater}^{5}$, J I Geh ${ }^{6}$, D Cunningham ${ }^{7}, J_{\text {Blazeby }}^{8}$, R Roy $^{9}$, T Maughan $^{10}, \mathrm{G} \mathrm{Griffiths}^{11}$ and S Mukherjee ${ }^{10}$

${ }^{1}$ Velindre Cancer Centre, Velindre Hospital, Cardiff CF14 2TL, UK; ${ }^{2}$ Wales Cancer Trials Unit, Cardiff University, Cardiff CF14 4YS, UK; ${ }^{3}$ Bristol Haematology and Oncology Centre, University Hospitals Bristol NHS Foundation Trust, Bristol BS2 8ED, UK; ${ }^{4}$ North Wales Cancer Treatment Centre, Conwy and Denbighshire NHS Trust, Rhyl LL18 5UJ, UK; ${ }^{5}$ UCL Cancer Institute, University College London, London WC1E 6BT, UK; ${ }^{6}$ Queen Elizabeth Hospital, University Hospitals Birmingham NHS Foundation Trust Birmingham B15 2GW, UK; ${ }^{7}$ The Royal Marsden Hospital NHS Foundation Trust, London SM2 5PT, UK; ${ }^{8}$ Centre for Surgical Research, University of Bristol, Bristol BS8 2PS, UK; ${ }^{9}$ Diana Princess of Wales Hospital, Northern Lincolnshire and Goole NHS Foundation Trust, Grimsby DN33 2BA, UK; ${ }^{10}$ CRUK/MRC Oxford Institute for Radiation Oncology, University of Oxford, Oxford OX3 7DQ, UK and ${ }^{11}$ Southampton Clinical Trials Unit, University of Southampton, Southampton SO16 6YD, UK

Background: The SCOPE-1 study tested the role of adding cetuximab to conventional definitive chemoradiotherapy (dCRT), and demonstrated greater toxicity and worse survival outcomes. We present the long-term outcomes and patterns of recurrence.

Methods: SCOPE-1 was a phase II/III trial in which patients were randomised to cisplatin $60 \mathrm{mg} \mathrm{m}^{-2}$ (day 1) and capecitabine $625 \mathrm{mg} \mathrm{m}^{-2}$ bd (days 1-21) for four cycles +1 - cetuximab $400 \mathrm{mg} \mathrm{m}^{-2}$ day 1 then by $250 \mathrm{mg} \mathrm{m}^{-2}$ weekly. Radiotherapy consisted of 50 Gy/25\# given concurrently with cycles 3 and 4. Recruitment was between February 2008 and February 2012, when the IDMC recommended closure on the basis of futility.

Results: About 258 patients $(d C R T=129 ; d C R T+$ cetuximab $(d C R T+C)=129)$ were recruited from 36 centres. About $72.9 \%$ $(n=188)$ had squamous cell histology. The median follow-up (IQR) was 46.2 (35.9-48.3) months for surviving patients. The median overall survival (OS; months; 95\% Cl) was 34.5 (24.7-42.3) in dCRT and 24.7 (18.6-31.3) in dCRT + C (hazard ratio (HR) $=1.25,95 \%$ Cls: 0.93-1.69, $P=0.137)$. Median progression-free survival (PFS; months; $95 \% \mathrm{Cl}$ ) was 24.1 (15.3-29.9) and 15.9 (10.7-20.8) months, respectively $(H R=1.28,95 \% C l s: 0.94-1.75 ; P=0.114)$. On multivariable analysis only earlier stage, full-dose $R T$, and higher cisplatin dose intensity were associated with improved OS.

Conclusions: The mature analysis demonstrates that the dCRT regimen used in the study provided useful survival outcomes despite its use in patients who were largely unfit for surgery or who had inoperable disease. Given the competing risk of systemic and local failure, future studies should continue to focus on enhancing local control as well as optimising systemic therapy.

Definitive chemoradiotherapy (dCRT) is an important treatment option for localised oesophageal cancer, and is considered a standard of care for patients with oesophageal squamous cell carcinoma. Long-term outcomes from dCRT, from large prospective clinical trials incorporating a modern conformal radiotherapy delivery protocol, QOL, and a robust radiotherapy quality 
assurance programme (RTQA) have not previously been reported. This information is important to allow patients to make an informed choice regarding treatment options, especially as nonrandomised data show that QOL may return to normal more quickly following dCRT and is better in the longer term compared with surgery (Rees et al, 2015).

Cancer Research UK SCOPE-1 trial was a randomised control phase II/III trial, which compared conventional cisplatincapecitabine-based dCRT with or without addition of cetuximab. The addition of cetuximab to radiotherapy for head and neck cancer had previously shown a significant survival benefit (Bonner et al, 2006). Following advice from the Independent Data Monitoring Committee (IDMC), SCOPE-1 was stopped at the phase II stage after 258 patients were randomised from 36 centres across the UK between February 2008 and January 2012, because the trial met the criteria for futility. The initial publication in 2013, after a median follow-up of 16.8 months in surviving patients (IQR 11.2-24.5), reported a statistically significant overall survival (OS) detriment in the cetuximab arm (median 25.4 months (95\% CIs: 20.5-37.9) vs 22.1 months (15.1-24.5); adjusted hazard ratio (HR) 1.53 (95\% CI 1.03-2.27); $P=0.035$; Crosby et al, 2013).

In this final report, we have looked at the long-term outcomes in the SCOPE-1, with detailed analysis of secondary end points, patterns of failure, and implications for future research. ISRCTN: 47718479.

\section{MATERIALS AND METHODS}

The trial design, treatments, eligibility criteria, and follow-up were previously reported in detail (Hurt et al, 2011; Crosby et al, 2013). In summary, the trial included patients with non-metastatic, histologically confirmed carcinoma of the oesophagus (adenocarcinoma, squamous cell, or undifferentiated; WHO status $0-1$; stage I-III disease; American Joint Committee on Cancer, 6th edition) and who had been selected to receive dCRT and randomly assigned them to receive dCRT alone or dCRT with cetuximab ( $400 \mathrm{mg} \mathrm{m}^{-2}$ on day 1 followed by $250 \mathrm{mg} \mathrm{m}^{-2}$ weekly). Definitive chemoradiotherapy consisted of cisplatin $60 \mathrm{mg} \mathrm{m}^{-2}$ (day 1) and capecitabine $625 \mathrm{mg} \mathrm{m}^{-2}$ bd twice daily (days 1-21) for four cycles; cycles 3 and 4 were given concurrently with 50 Gy in 25 fractions of radiotherapy. Follow-up was at 24 weeks, then every 3 months during the first year, every 4 months during the second year, and yearly thereafter for a minimum of 5 years from randomisation, during which time RTOG/EORTC late radiation morbidity scores (Radiation Therapy Oncology Group, 2007) were collected. The primary end point was the proportion of patients with treatment failure-free survival (TFFS, defined as still alive with no evidence of residual malignancy in the endoscopic biopsy sample and no evidence of disease progression outside the radiotherapy field on CT scan) at week 24 for the phase II stage and OS (time to event) for the phase III stage of the trial, both of which were measured from randomisation. Secondary end points included local and distant progression-free survival (LPFS and DPFS), patterns of first progression, and late toxicity. Distant progression-free survival was defined as time to progression with metastases or death by any cause. Local progression-free survival was defined as either time to progression within the RT volume (infield) or death by any cause, or outside the RT volume but within the region (outfield) or death by any cause. QOL data were collected up to 24 months and have been reported elsewhere (Rees et al, 2015). The trial was approved by a UK multi-centre ethics committee and obtained individual informed consent from all participants. The trial protocol and radiotherapy guideline can be found in Supplementary Materials 1 and 2, respectively.

All statistical analyses were pre-planned and conducted using Stata SE 14 (StataCorp, College Station, TX, USA). All analyses were performed according to the intention-to-treat principle and included all randomly assigned patients. Follow-up time distributions were estimated using the reverse Kaplan-Meier method (Schemper and Smith, 1996) with patients censored at date of death or last trial assessment. We calculated \% of total dose (actual total dose divided by protocol total dose) and \% dose intensity (actual dose intensity (dose per unit time) divided by protocol dose intensity) for each protocol drug as measures of compliance. As has been done elsewhere (Loibl et al, 2011), patients who progressed or died during the treatment period had denominators calculated up to the point where they progressed or died. Likewise for radiotherapy we calculated \% of full protocol dose received by each patient and for those who progressed or died during the treatment period, the denominator was calculated up to the point where they progressed or died. We calculated survival from date of randomisation to when an event occurred, that is, progression or any death for PFS, and any death for OS. Patients who were event free were censored at the time they were last known to be event free. We estimated event time distributions with the Kaplan-Meier method and compared OS and PFS with HRs from Cox regression in univariable models and multivariable models. In the multivariable models we included all variables thought a priori to potentially have a prognostic effect and included recruitment centre as a random frailty effect. The consistency of the main univariable treatment arm effect was assessed across subgroups using HR plots and the significance of treatment arm-subgroup variable interaction terms in Cox models. We tested the proportional hazards assumption of each model with Cox-Snell residuals and Schoenfeld's global test. We did not adjust for having previously looked at the HRs, as survival was a secondary end point to the phase II trial.

\section{RESULTS}

Study population. The study population was described in detail in the first report and Figure 1; (Crosby et al, 2013). In summary, median age was 66.7 (IQR: 60.9-72.9), 56.2\% $(n=145)$ were male,

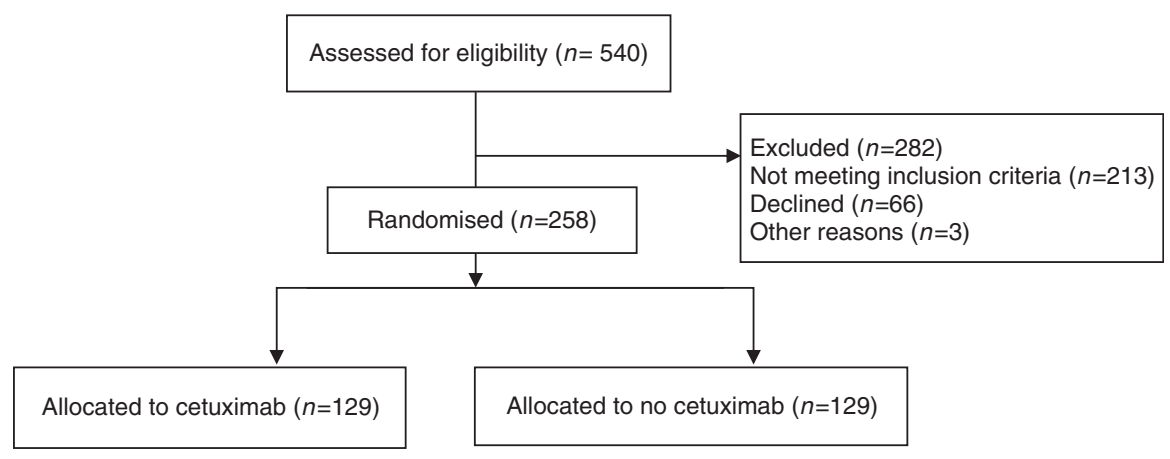

Figure 1. Flow diagram of trial participants. 
$50.8 \%(n=131)$ were WHO PS 0 (rather than 1$), 60.1 \%(n=155)$ had stage III disease, $72.9 \%(n=188)$ had squamous cell type, and the median disease length was $5 \mathrm{~cm}$ (IQR: 4-7.5). The main reason for no surgery was local extent of disease $(47.3 \%, n=122)$. T stage: T1 3.5\% $(n=9)$, T2 $18.2 \%(n=47)$, T3 63.2\% $(n=163)$, T4 $15.1 \%$ $(n=39)$. N stage: N0 $33.3 \%(n=86), \mathrm{N} 166.7 \%(n=172)$.

Median follow-up was 46.2 (IQR: 35.9-48.3) months for surviving patients. This was balanced across trial arms with median times of 45.2 (IQR: 35.8-48.2) and 46.8 (IQR: 36.4-48.8) months in the dCRT only and dCRT $+\mathrm{C}$ arms, respectively (Figure $2 \mathrm{~A}$ ).

A

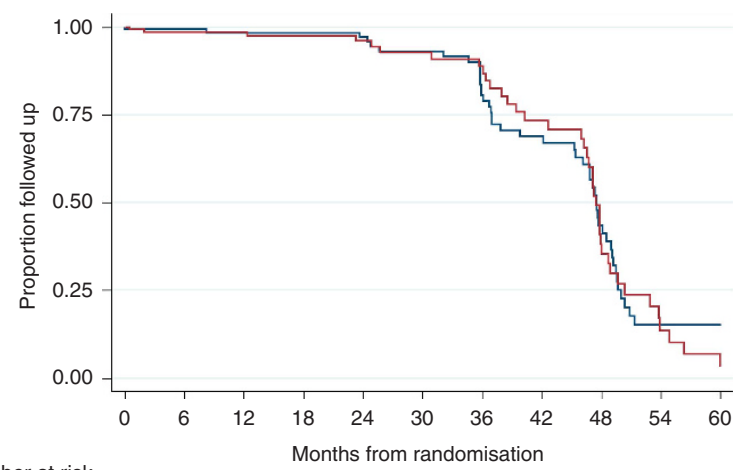

Number at risk

$\begin{array}{llllllllllll}\text { dCRT } & 129 & 120 & 105 & 89 & 75 & 63 & 49 & 38 & 19 & 6 & 3\end{array}$ $\begin{array}{llllllll}\mathrm{dCRT}+\mathrm{C} & 129 & 111 & 92 & 77 & 63 & & \\ & & & & & \end{array}$

C

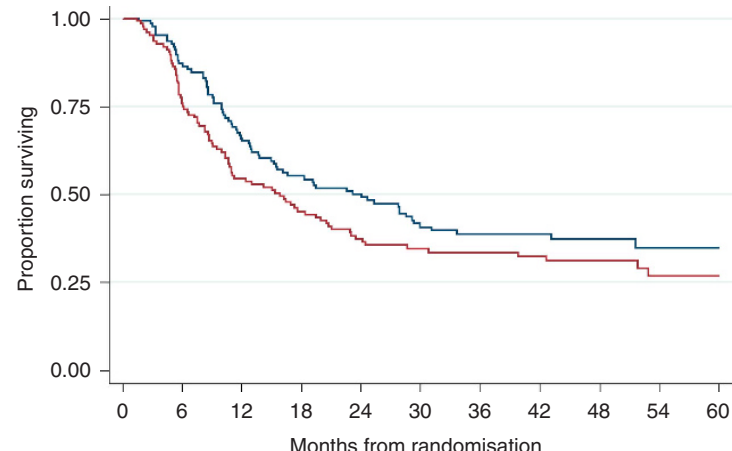

Number at risk

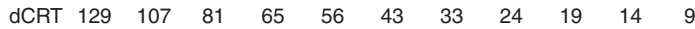
$\begin{array}{llllllllllll}\mathrm{dCRT}+\mathrm{C} & 129 & 94 & 65 & 53 & 42 & 34 & 32 & 26 & 17 & 12 & 8\end{array}$

E

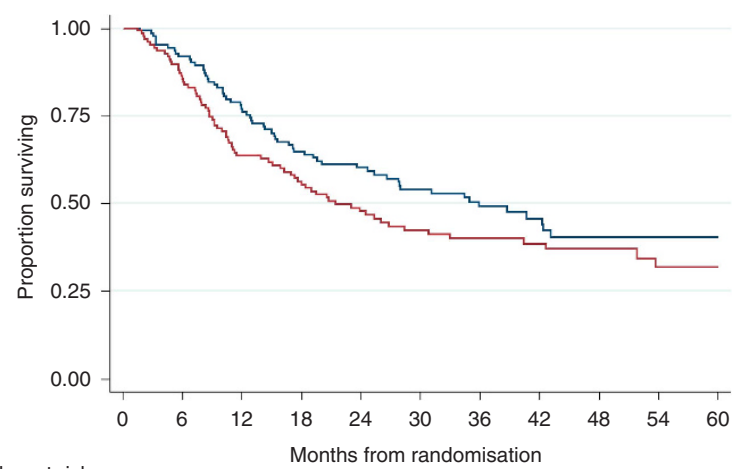

Number at risk

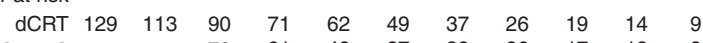

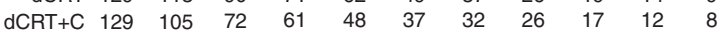

Compliance. Significantly more patients completed all four cycles of cisplatin and capecitabine in the dCRT-only group (Crosby et al, 2013 ). More patients in the dCRT group received $\geqslant 75 \%$ of the total protocol dose of capecitabine (72.1 (93 out of 129) vs $62.8 \%$ (81 out of 129$), \chi^{2}=2.542, P=0.111$ ). The proportion of patients receiving $\geqslant 75 \%$ of the full protocol cisplatin dose intensity was similar in both trial arms (dCRT: 71\% (91 out of 129); dCRT + C: $71 \%$ (92 out of 129); Supplementary Figure S1). This was mainly due to a larger proportion of patients in the $\mathrm{dCRT}+\mathrm{C}$ group receiving full cisplatin dose in the second cycle of chemotherapy.

B

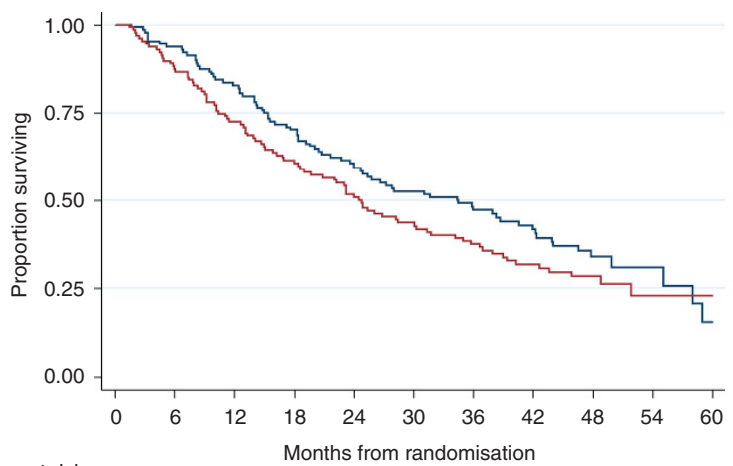

Number at risk

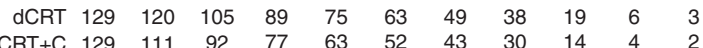

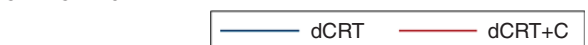

D

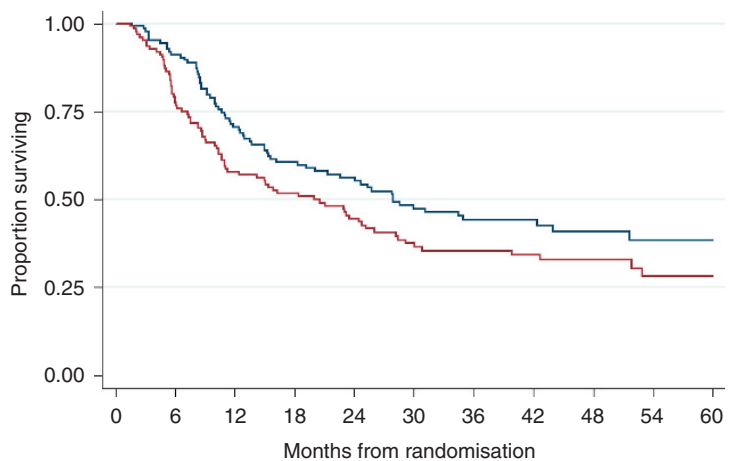

Number at risk

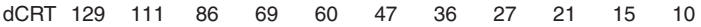

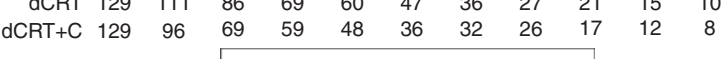

$\mathbf{F}$

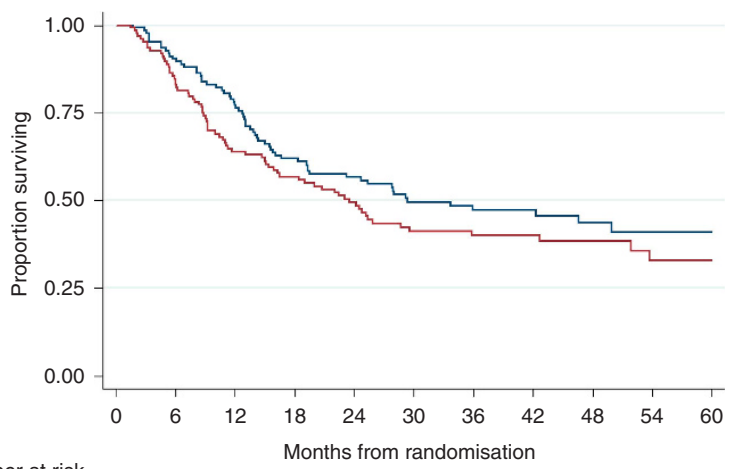

Number at risk

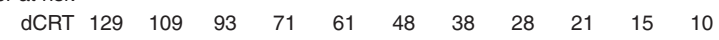

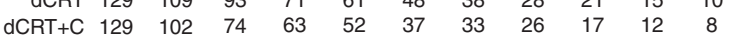

Figure 2. Kaplan-Meier curves of follow-up and survival by treatment group. (A) Follow-up (reverse Kaplan-Meier). (B) Overall survival. (C) Progression-free survival. (D) Local progression-free survival (infield). (E) Local progression-free survival (outfield). (F) Distant progression-free survival. 
Significantly more patients in the dCRT group received the full protocol dose of radiotherapy (90.7 (117 out of 129) vs 79.1\% (102 out of 129$), \chi^{2}=6.796, P=0.009$ ). Six patients died (three due to oesophageal cancer, one sepsis, one stroke, and one vascular disorder of intestine) prior to the end of the treatment period.

Toxicities. The RTOG/EORTC late radiation morbidity scores are shown in Supplementary Table S1. Assessment completion rates were high with scores being obtained in between $91.5 \%$ (214 out of $234)$ at 6 months and $87.9 \%$ (123 out of 140) at 24 months. Rates of oesophageal late radiation morbidity were initially slightly higher in the dCRT arm reflecting the higher doses achieved in that arm. However, across arms the rates of worst grade of any residual toxicity were: $9.3 \%$ (20 out of 214 ) grade $1,6.5 \%$ (14 out of 214 ) grade 2 , and $0.5 \%$ ( 1 out of 214 ) grade 3 at 6 months and this dropped to only $2.4 \%$ ( 3 out of 123 ) grade 1 at 24 months.

Overall survival. The mature OS analysis (Table 1 and Figure 2B) demonstrates the median survival in the dCRT arm to be 34.5 months (95\% CI: 24.7-42.3) vs 24.7 months (95\% CI: 18.6-31.3) in the dCRT $+\mathrm{C}$ arm. With prolonged follow-up, the HR was no longer statistically significant in either univariable or multivariable analysis (1.25 (95\% CIs: 0.93-1.69, $P=0.137)$ and 1.15 (95\% CIs: 0.84-1.57,
$P=0.388$ ), respectively). Three-year OS was $47.2 \%$ (95\% CIs: $38.2-$ $55.7)$ in the dCRT-only arm and $37.6 \%$ (95\% CIs: $29.1-46.0)$ in the $\mathrm{dCRT}+\mathrm{C}$ arm. In patients receiving conventional dCRT: with squamous cell subtype, the median OS was 35.9 months (95\% CI: 24.7-44.0; $n=96$, 3-year OS: $47.8 \%$ (95\% CI: $37.1-57.6 \%)$ ); in the adenocarcinoma subtype the median OS was 25.8 months (95\% CIs: 12.5-46.6; $n=32$; 3-year OS: $43.8 \%$ (95\% CIs: 26.5-59.8\%)).

Higher stage, less than full protocol radiotherapy dose, and lower cisplatin dose intensity were associated with worse survival in multivariable analysis (Table 1). The better survival in the dCRT arm was consistent across most subgroups of baseline characteristics other than in females and those with disease length $\geqslant 8 \mathrm{~cm}$ (Figure 3 ) and only the sex treatment arm interaction term was significant in Cox models $(z=2.58, P=0.010)$. In females the HR for treatment effect was 0.81 (95\% CI: 0.50-1.31), whereas in males it was 1.87 (95\% CIs: $1.26-2.77$ ). A sensitivity analysis that only included patients who were alive at the end of the treatment period gave the same findings.

TTFS at 24 weeks continued to be highly prognostic of OS with the failures $(n=68)$ having a median OS of 8.3 months (95\% CIs: $6.7-12.5)$ and those failure free $(n=172)$ having a median OS of 42.3 months (95\% CIs: 35.9-48.8).

Table 1. Univariable and multivariable Cox regression analysis of OS

\begin{tabular}{|c|c|c|c|c|c|c|c|c|c|}
\hline & \multicolumn{3}{|c|}{ OS (months) } & \multicolumn{3}{|c|}{ Univariable } & \multicolumn{3}{|c|}{ Multivariable } \\
\hline & $n$ & Median & $95 \% \mathrm{Cls}$ & HR & $95 \% \mathrm{Cls}$ & $P$-value & HR & $95 \% \mathrm{Cls}$ & $P$-value \\
\hline \multicolumn{10}{|l|}{ Trial arm } \\
\hline CRT only & 129 & 34.5 & $24.7-42.3$ & 1 & & & 1 & & \\
\hline CRT + cetuximab & 129 & 24.7 & $18.6-31.3$ & 1.25 & $0.93-1.69$ & 0.137 & 1.15 & $0.84-1.57$ & 0.388 \\
\hline \multicolumn{10}{|l|}{ Age } \\
\hline$<65$ & 109 & 36.7 & $24.9-43.6$ & 1 & & & 1 & & \\
\hline$\geqslant 65$ & 149 & 24.5 & $19.7-30.1$ & 1.36 & $1.00-1.85$ & 0.047 & 1.32 & $0.95-1.84$ & 0.093 \\
\hline \multicolumn{10}{|c|}{ Reason for no surgery } \\
\hline Patient choice & 97 & 31.3 & $24.0-44.0$ & 1 & & & 1 & & \\
\hline Local extent of disease & 122 & 24.7 & $18.6-34.5$ & 1.2 & $0.86-1.68$ & 0.276 & 0.97 & $0.67-1.41$ & 0.875 \\
\hline Comorbidity/poor PS & 39 & 31.6 & $14.8-42.7$ & 1.25 & $0.81-1.94$ & 0.318 & 0.98 & $0.58-1.67$ & 0.949 \\
\hline \multicolumn{10}{|l|}{ Sex } \\
\hline Female & 113 & 34.6 & $24.7-48.8$ & 1 & & & 1 & & \\
\hline Male & 145 & 24.9 & $19.6-31.6$ & 1.44 & $1.06-1.95$ & 0.02 & 1.35 & $0.96-1.90$ & 0.083 \\
\hline \multicolumn{10}{|l|}{ WHO status } \\
\hline 0 & 131 & 30.3 & $24.0-38.4$ & 1 & & & 1 & & \\
\hline 1 & 127 & 24.9 & $19.2-34.3$ & 1.14 & $0.84-1.53$ & 0.405 & 1.07 & $0.77-1.50$ & 0.675 \\
\hline \multicolumn{10}{|l|}{ Stage } \\
\hline I or II & 103 & 42.4 & $31.3-49.9$ & 1 & & & 1 & & \\
\hline III & 155 & 24 & $18.6-26.8$ & 1.65 & $1.20-2.27$ & 0.002 & 1.52 & $1.05-2.21$ & 0.028 \\
\hline \multicolumn{10}{|l|}{ Tumor type } \\
\hline Squamous & 188 & 28.4 & $24.0-38.0$ & 1 & & & 1 & & \\
\hline Adeno/undiff & 70 & 24.9 & $15.9-35.1$ & 1.24 & $0.90-1.72$ & 0.192 & 1.01 & $0.67-1.52$ & 0.961 \\
\hline \multicolumn{10}{|l|}{ Full radiation dose } \\
\hline Yes & 217 & 34.3 & $25.8-39.1$ & 1 & & & 1 & & \\
\hline No & 41 & 10 & 5.9-18.4 & 3.19 & $2.17-4.70$ & 0 & 2.06 & $1.21-3.49$ & 0.008 \\
\hline \multicolumn{10}{|l|}{ Cisplatin intensity } \\
\hline$\geqslant 75 \%$ & 182 & 35.9 & $27.2-42.4$ & 1 & & & 1 & & \\
\hline$<75 \%$ & 76 & 16.2 & $12.5-20.8$ & 2.18 & 1.59-2.99 & 0 & 1.8 & $1.12-2.89$ & 0.016 \\
\hline \multicolumn{10}{|l|}{ Cape/5FU intensity } \\
\hline$\geqslant 75 \%$ & 172 & 34.5 & $25.4-39.4$ & 1 & & & 1 & & \\
\hline$<75 \%$ & 86 & 20 & $15.4-24.7$ & 1.66 & $1.22-2.26$ & 0.001 & 0.85 & $0.54-1.34$ & 0.493 \\
\hline \multicolumn{10}{|l|}{ Total disease length } \\
\hline$<4 \mathrm{~cm}$ & 56 & 36 & $24.7-58.0$ & 1 & & & 1 & & \\
\hline$\geqslant 4-<6 \mathrm{~cm}$ & 85 & 37.9 & $24.0-49.9$ & 0.98 & $0.63-1.52$ & 0.928 & 0.98 & $0.62-1.55$ & 0.928 \\
\hline$\geqslant 6-<8 \mathrm{~cm}$ & 55 & 24.9 & $18.6-40.3$ & 1.46 & $0.92-2.33$ & 0.107 & 1.2 & $0.72-2.00$ & 0.489 \\
\hline$\geqslant 8 \mathrm{~cm}$ & 62 & 18.4 & $14.9-27.8$ & 1.84 & $1.17-2.89$ & 0.009 & 1.5 & $0.91-2.50$ & 0.115 \\
\hline
\end{tabular}




$\begin{array}{lrr}\text { Age } & n & \text { Deaths } \\ <65 & 109 & 68 \\ 65 \text { and over } & 149 & 106 \\ & & \\ \text { Reason for no surgery } & 97 & 63 \\ \text { Patient choice } & 122 & 81 \\ \text { Local extent of disease } & 39 & 30 \\ \text { Comorbidity/poor performance status } & & \\ \text { Sex } & 113 & 67 \\ \text { Female } & 145 & 107 \\ \text { Male } & & \\ \text { WHO performance status } & 131 & 88 \\ 0 & 127 & 86 \\ 1 & & \\ \text { Tumour type } & 188 & 120 \\ \text { Squamous cell } & 70 & 54 \\ \text { Adenocarcinoma/undifferentiated } & & \\ \text { Stage } & 103 & 61 \\ \text { Stage I/II } & 155 & 113 \\ \text { Stage III } & & \\ \text { Total disease length } & 56 & 34 \\ <4 \mathrm{~cm} & 85 & 50 \\ 4-<6 \mathrm{~cm} & 55 & 42 \\ 6-<8 \mathrm{~cm} & 62 & 48 \\ \geqslant 8 \mathrm{~cm} & & 0.5 \\ & & \end{array}$

Figure 3. Hazard ratio plots for treatment effect on overall survival by baseline characteristics (HR>1 favours dCRT only).

In the $\mathrm{dCRT}+\mathrm{C}$ arm only, there was no difference in survival between those with any grade $3+$ dermatological toxicity during treatment $(n=28)$ when compared to those without $(n=101)$ $(\mathrm{HR}=1.03,95 \% \mathrm{CI}: 0.63-1.69, P=0.895)$.

Progression-free survival. The PFS analysis (Table 2 and Figure 2C) suggests that the median PFS was still higher in the dCRT-only arm (24.1 months (95\% CIs: 15.3-29.9) vs 15.9 months (95\% CIs: 10.7-20.8)), although the HR was still not significant on either univariable or multivariable analysis $(\mathrm{HR}=1.28$; $95 \%$ CIs: $0.94-1.75 ; P=0.114$ and $\mathrm{HR}=1.20$; $95 \%$ CIs: $0.87-1.66 ; P=0.220$, respectively). Higher stage, male sex, and lower cisplatin dose intensity were associated with worse PFS in multivariable analysis. A sensitivity analysis that only included patients who were alive at the end of the treatment period gave the same findings.

Similar results were seen in LPFS (infield), LPFS (outfield), and DPFS (Figure 2D-F). The median LPFS (infield) was better in the dCRT arm and this approached statistical significance: 27.9 months (95\% CIs: 19.2-51.7) vs 20.0 months (95\% CIs: 11.1-26.1), HR = 1.36 (95\% CIs: $0.99-1.87, P=0.062$ ). Differences in LPFS (outfield) and DPFS also favoured the dCRT arm although with no statistical significance; median LPFS (outfield): 35.9 months (24.7-62.1) vs 21.5 months (16.3-33.0), $\mathrm{HR}=1.32$ (0.94-1.85), $P=0.112$; median DPFS: 29.3 months (19.3-60.6) vs 23.5 months (15.9-35.8), HR $=1.24(0.89-1.73), P=0.207$. In the multivariable models for LPFS (infield), LPFS (outfield), and DPFS, only less than full protocol radiotherapy dose and lower cisplatin dose intensity remained significant at the $P<0.05$ level (data not shown).

Patterns of first progression. The patterns of first progression are shown in Table 3 . There were 77 progressions prior to death or date last seen in the dCRT arm and 85 in the dCRT $+\mathrm{C}$ group. More progressions involved a distant progression only (76 out of $162(46.9 \%))$ compared to loco-regional progression only (57 out of $162(35.2 \%))$. In the dCRT arm, out of 38 patients with a locoregional progression, 31 progressed within the RT field compared to 40 out of 48 in the dCRT $+C$ group $(81.6 \%$ vs $83.3 \%$, respectively, $\left.\chi^{2}=0.0453, P=0.831\right)$. Very similar patterns were seen in both squamous cell and adenocarcinoma/undifferentiated tumours (Supplementary Table S2).

Causes of death. At the time of analysis, 84 (65.1\%) patients had died (69 (53.5\%) of oesophageal cancer) in the dCRT arm and 90 $(69.8 \%)$ patients had died (78 $(60.5 \%)$ of oesophageal cancer) in the dCRT $+\mathrm{C}$ arm (Supplementary Table S3).

\section{DISCUSSION}

These long-term data, analysed after death of nearly two-thirds of the patients in both arms and after a median follow-up of 46.2 months in surviving patients, show that median OS in the dCRT arm (34.5 months (95\% CI: $24.7-42.3)$ ) is better than initially reported. Although survival in the standard dCRT arm remained superior to the cetuximab arm, the difference was no longer statistically significant. Local progression within radiation field was higher in the cetuximab arm, which approached statistical significance $(P=0.062)$; full-dose radiotherapy and a cisplatin dose intensity of $>75 \%$ were associated with improved survival in the multivariable model. Long-term residual treatment toxicity was very low and TFFS remained a highly significant surrogate for OS. A difference in treatment effect was found by sex with the better survival in the dCRT arm not being seen in females; however, this was an exploratory analysis for which we do not have a hypothesis and may be due to chance, therefore further corroboration is needed (Wallach et al, 2016).

At the time of initiating this study, there was significant interest in combining radiotherapy with cetuximab following reported survival advantage for this combination in a randomised controlled trial in head and neck cancer, which showed near doubling of median OS (54 vs 28 months, $P=0.02)$ and improved 3-year OS (57 vs 44\%; Bonner et al, 2006). Preclinical data suggested radioresistance in EGFR overexpressing head and neck cell lines and this radioresistance could be reversed through EGFR blockade (Akimoto et al, 1999; Huang et al, 1999, 2002; Bonner et al, 2000; Milas et al, 2000, 2004; Harari and Huang, 2001; Nasu et al, 2001; Shintani et al, 2003). Subsequent to SCOPE-1, several other studies have reported outcomes from EGFR inhibition and CRT in oesophageal cancer (Ruhstaller et al, 2011; Becerra et al, 2013; Meng et al, 2013; Ubink et al, 2014). Most notably, the RTOG 0436 trial randomised 344 patients to weekly paclitaxel, cisplatin with or without cetuximab and $50.4 \mathrm{~Gy}$ of radiotherapy. The study failed to demonstrate improvements in OS, local control, or clinical complete response rate (Suntharalingam et al, 2014).

The inability to deliver adequate doses of standard chemoradiotherapy treatment has been a consistent feature of randomised trials of cetuximab in head and neck, and GI cancer which may have contributed to inferior outcomes in the cetuximab arms (Maughan et al, 2011; Waddell et al, 2013; Ang et al, 2014). In RTOG 0522, a trial designed to test the benefits for the addition of cetuximab to chemoradiotherapy in squamous cell head and neck cancer, patients receiving cetuximab experienced more interruptions to radiotherapy treatment and no survival advantage. The findings from the SCOPE1 trial are consistent with these observations.

Despite the lack of benefit from the addition of cetuximab, it is important to report long-term outcomes of dCRT trials, as mature survival data using this modality in oesophageal cancer are lacking. Although a significant proportion of patient was elderly $(39 \%$ above the age of 70 years), the majority had stage 3 disease (60\%), and most patients were unsuitable for surgery due to advanced local disease (47\%) and/or co-morbidities (16\%); the OS in the standard arm of this study is among the best in published literature on dCRT (Cooper et al, 1999; Stahl et al, 2005; Bedenne et al, 2007; Herskovic et al, 2012; Conroy et al, 2014). The encouraging 
Table 2. Univariable and multivariable Cox regression analysis of PFS

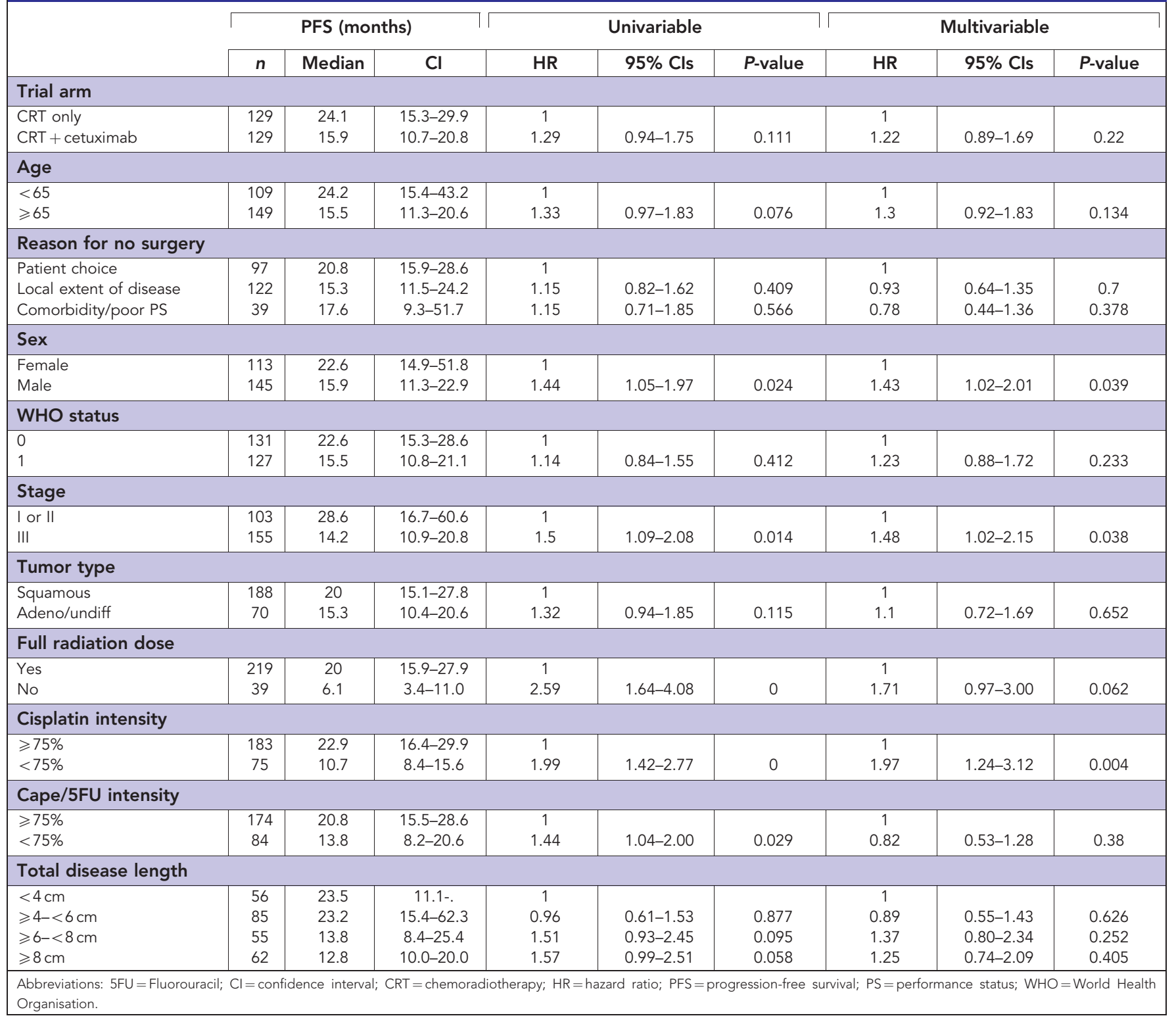

long-term survival seen in SCOPE-1 reassures us that dCRT is not an unreasonable alternative to surgery, particularly in patients with borderline fitness where surgery is considered a high-risk procedure. In addition, local recurrence, often raised as an important weakness of dCRT over surgery, was lower in this trial when compared with other historic reports of dCRT (Minsky et al, 2002; Denham et al, 2003; Herskovic et al, 2012).

So what factors are likely to have contributed to the good outcome seen in the dCRT arm of this trial compared to historic studies? For the first time in the United Kingdom, this trial introduced a detailed protocol for staging patients with oesophageal cancer due to undergoing non-surgical treatment, thereby improving patient selection - all eligible patients had an EUS and $85 \%$ were staged with $18 \mathrm{~F}-\mathrm{FDG}$ CT-PET. It also introduced what, at the time, was advanced, conformal radiotherapy treatment by way of a detailed radiotherapy planning guidance document together with a RTQA programme (Gwynne et al, 2013). The authors believe such RTQA programmes, which included pre-trial test case and real-time radiotherapy planning reviews in a proportion of patients, would have contributed to the improved outcomes and should become a mandatory component of future radiotherapy trials (Ibbott et al, 2013).
Treatments after the end of trial chemoradiotherapy were not accounted for in the analysis and therefore the impact of secondand subsequent-line therapies on OS cannot be ascertained. Moreover, no imaging was mandated after 24 weeks and detection of progression relied on clinical assessment at follow-up visits mandating serial imaging may have led to earlier detection of disease progression and therefore earlier institution of second-line treatment. Nevertheless, this mirrors standard of care for this patient population and there is little evidence for a survival advantage for any treatment intervention over another post dCRT.

The failure of SCOPE-1 and other cetuximab-based studies argues against the non-selective use of biological agents in oncology trials, highlighting the potential for harm and not just lack of efficacy. The use of a less toxic chemotherapy backbone may also allow incorporation of novel agents into dCRT regimens without compromising dose intensity of conventional treatment. The weekly carboplatin-/paclitaxel-based neo-adjuvant CRT regimen, as established by the CROSS trial Shintani et al, 2003, reported low incidence of treatment-related toxicity and an impressive pathological complete response of about $50 \%$ in the squamous cell subtype - such low-toxicity regimens should be explored in the context of dCRT trials in oesophageal cancer. 
Table 3. Patterns of first progression in relation to radiation target volumes (number of events) by trial arm

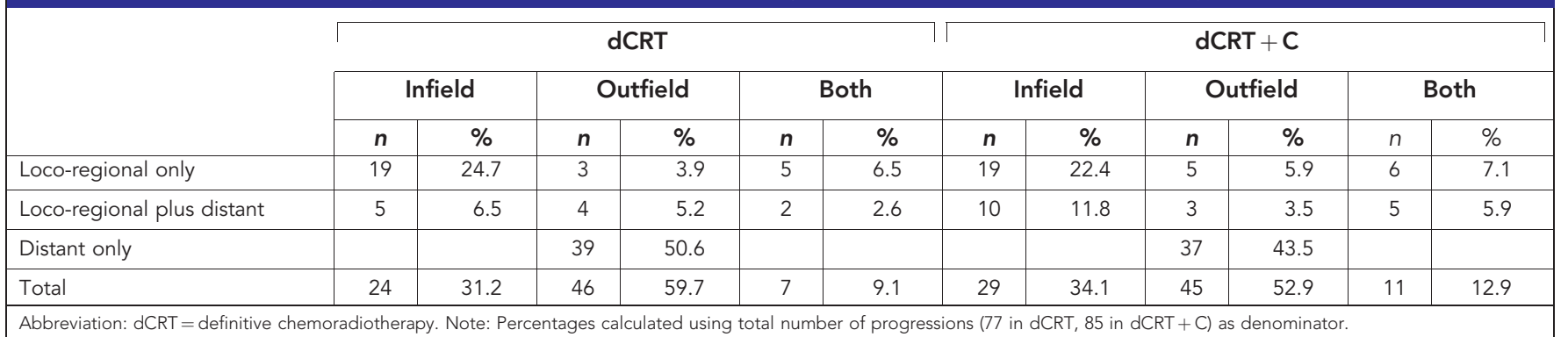

In summary, we concur with our previous report that cetuximab should not be used as standard with CRT in the treatment of oesophageal cancer. This mature analysis highlighted the excellent survival and low rate of long-term toxicity of standard dCRT, which taken in conjunction with the early return of quality of life (Rees et al, 2015), suggests dCRT is an effective treatment modality. Given the competing risks of systemic and local failure, efforts should continue to maximise local control through radiotherapy dose intensification (in the context of modern day radiotherapy planning and delivery) and to identify more effective systemic treatments for those patients who do not, or are unlikely to, respond to conventional dCRT. Such an approach, including radiotherapy dose escalation and individualisation of systemic therapy through early PET response will be pursued in Cancer Research UK-funded SCOPE-2 trial, currently in set up in the United Kingdom (EudraCT No: 2015-001740-11).

\section{ACKNOWLEDGEMENTS}

The SCOPE-1 trial was sponsored by Velindre NHS Trust. We thank all the patients who participated in the trial, the doctors, nurses, pathologists, and other members of the MDTs; the radiotherapy team and the research team from the participating centres. We would like to thank members of the Independent Data Monitoring Committee and the Independent Trial Steering Committee for their oversight of the trial. This work was supported by Cancer Research UK (grant number C20177/A7256) and Cancer Research UK core funding at Wales Cancer Trials Unit. Merck provided free cetuximab, labelling, and distribution for the study. The RTTQA was funded by Cancer Research UK and the Cardiff NCRI RTTQA Center at Velindre NHS Trust who are funded by the Health and Social Care Research Wales, and Department of Health (England). SM is funded by NIHR Oxford Biomedical Research Centre and the CRUK Experimental Cancer Medicines Centre, Oxford.

\section{CONFLICT OF INTEREST}

The authors declare no conflict of interest.

\section{REFERENCES}

Akimoto T, Hunter NR, Buchmiller L, Mason K, Ang KK, Milas L (1999) Inverse relationship between epidermal growth factor receptor expression and radiocurability of murine carcinomas. Clin Cancer Res 5(10): 2884-2890.

Ang KK, Zhang Q, Rosenthal DI, Nguyen-Tan PF, Sherman EJ, Weber RS, Galvin JM, Bonner JA, Harris J, El-Naggar AK, Gillison ML, Jordan RC, Konski AA, Thorstad WL, Trotti A, Beitler JJ, Garden AS, Spanos WJ, Yom SS, Axelrod RS (2014) Randomized phase III trial of concurrent accelerated radiation plus cisplatin with or without cetuximab for stage III to IV head and neck carcinoma: RTOG 0522. J Clin Oncol 32(27): 2940-2950.

Becerra CR, Hanna N, McCollum AD, Becharm N, Timmerman RD, DiMaio M, Kesler KA, Yu M, Yan T, Choy H (2013) A phase II study with cetuximab and radiation therapy for patients with surgically resectable esophageal and GE junction carcinomas: Hoosier Oncology Group G05-92. J Thorac Oncol 8(11): 1425-1429.

Bedenne L, Michel P, Bouche O, Milan C, Mariette C, Conroy T, Pezet D, Roullet B, Seitz JF, Herr JP, Paillot B, Arveux P, Bonnetain F, Binquet C (2007) Chemoradiation followed by surgery compared with chemoradiation alone in squamous cancer of the esophagus: FFCD 9102. J Clin Oncol 25(10): 1160-1168.

Bonner JA, Harari PM, Giralt J, Azarnia N, Shin DM, Cohen RB, Jones CU, Sur R, Raben D, Jassem J, Ove R, Kies MS, Baselga J, Youssoufian H, Amellal N, Rowinsky EK, Ang KK (2006) Radiotherapy plus cetuximab for squamous-cell carcinoma of the head and neck. $N$ Engl J Med 354(6): 567-578.

Bonner JA, Raisch KP, Trummell HQ, Robert F, Meredith RF, Spencer SA, Buchsbaum DJ, Saleh MN, Stackhouse MA, LoBuglio AF, Peters GE, Carroll WR, Waksal HW (2000) Enhanced apoptosis with combination C225/radiation treatment serves as the impetus for clinical investigation in head and neck cancers. J Clin Oncol 18(21 Suppl): 47S-53S.

Conroy T, Galais MP, Raoul JL, Bouche O, Gourgou-Bourgade S, Douillard JY, Etienne PL, Boige V, Martel-Lafay I, Michel P, Llacer-Moscardo C, Francois E, Crehange G, Abdelghani MB, Juzyna B, Bedenne L, Adenis A, Federation Francophone de Cancerologie D, Group U-G (2014) Definitive chemoradiotherapy with FOLFOX versus fluorouracil and cisplatin in patients with oesophageal cancer (PRODIGE5/ACCORD17): final results of a randomised, phase 2/3 trial. Lancet Oncol 15(3): 305-314.

Cooper JS, Guo MD, Herskovic A, Macdonald JS, Martenson Jr JA, Al-Sarraf M, Byhardt R, Russell AH, Beitler JJ, Spencer S, Asbell SO, Graham MV, Leichman LL (1999) Chemoradiotherapy of locally advanced esophageal cancer: long-term follow-up of a prospective randomized trial (RTOG 85-01). Radiation Therapy Oncology Group. JAMA 281(17): $1623-1627$

Crosby T, Hurt CN, Falk S, Gollins S, Mukherjee S, Staffurth J, Ray R, Bashir N, Bridgewater JA, Geh JI, Cunningham D, Blazeby J, Roy R, Maughan T, Griffiths G (2013) Chemoradiotherapy with or without cetuximab in patients with oesophageal cancer (SCOPE1): a multicentre, phase 2/3 randomised trial. Lancet Oncol 14(7): 627-637.

Denham JW, Steigler A, Kilmurray J, Wratten C, Burmeister B, Lamb DS, Joseph D, Delaney G, Christie D, Jamieson G, Smithers BM, Ackland S, Walpole E (2003) Relapse patterns after chemo-radiation for carcinoma of the oesophagus. Clin Oncol (R Coll Radiol) 15(3): 98-108.

Gwynne S, Spezi E, Sebag-Montefiore D, Mukherjee S, Miles E, Conibear J, Staffurth J, Ncri Rttqa O, Imaging S (2013) Improving radiotherapy quality assurance in clinical trials: assessment of target volume delineation of the pre-accrual benchmark case. Br J Radiol 86: 20120398.

Harari PM, Huang SM (2001) Head and neck cancer as a clinical model for molecular targeting of therapy: combining EGFR blockade with radiation. Int J Radiat Oncol Biol Phys 49(2): 427-433.

Herskovic A, Russell W, Liptay M, Fidler MJ, Al-Sarraf M (2012) Esophageal carcinoma advances in treatment results for locally advanced disease: review. Ann Oncol 23(5): 1095-1103.

Huang SM, Bock JM, Harari PM (1999) Epidermal growth factor receptor blockade with C225 modulates proliferation, apoptosis, and radiosensitivity in squamous cell carcinomas of the head and neck. Cancer Res 59(8): 1935-1940. 
Huang SM, Li J, Armstrong EA, Harari PM (2002) Modulation of radiation response and tumor-induced angiogenesis after epidermal growth factor receptor inhibition by ZD1839 (Iressa). Cancer Res 62(15): 4300-4306.

Hurt CN, Nixon LS, Griffiths GO, Al-Mokhtar R, Gollins S, Staffurth JN, Phillips CJ, Blazeby JM, Crosby TD (2011) SCOPE1: a randomised phase II/III multicentre clinical trial of definitive chemoradiation, with or without cetuximab, in carcinoma of the oesophagus. BMC Cancer 11: 466.

Ibbott GS, Haworth A, Followill DS (2013) Quality assurance for clinical trials. Front Oncol 3: 311.

Loibl S, Skacel T, Nekljudova V, Luck HJ, Schwenkglenks M, Brodowicz T, Zielinski C, von Minckwitz G (2011) Evaluating the impact of relative total dose intensity (RTDI) on patients' short and long-term outcome in taxane- and anthracycline-based chemotherapy of metastatic breast cancer- a pooled analysis. BMC Cancer 11: 131.

Maughan TS, Adams RA, Smith CG, Meade AM, Seymour MT, Wilson RH, Idziaszczyk S, Harris R, Fisher D, Kenny SL, Kay E, Mitchell JK, Madi A, Jasani B, James MD, Bridgewater J, Kennedy MJ, Claes B, Lambrechts D, Kaplan R, Cheadle JP, Investigators MCT (2011) Addition of cetuximab to oxaliplatin-based first-line combination chemotherapy for treatment of advanced colorectal cancer: results of the randomised phase 3 MRC COIN trial. Lancet 377(9783): 2103-2114.

Meng X, Wang J, Sun X, Wang L, Ye M, Feng P, Zhu G, Lu Y, Han C, Zhu S, Liao Z, Yu J (2013) Cetuximab in combination with chemoradiotherapy in Chinese patients with non-resectable, locally advanced esophageal squamous cell carcinoma: a prospective, multicenter phase II trial. Radiother Oncol 109(2): 275-280.

Milas L, Fan Z, Andratschke NH, Ang KK (2004) Epidermal growth factor receptor and tumor response to radiation: in vivo preclinical studies. Int $J$ Radiat Oncol Biol Phys 58(3): 966-971.

Milas L, Mason K, Hunter N, Petersen S, Yamakawa M, Ang K, Mendelsohn J, Fan Z (2000) In vivo enhancement of tumor radioresponse by C225 antiepidermal growth factor receptor antibody. Clin Cancer Res 6(2): 701-708.

Minsky BD, Pajak TF, Ginsberg RJ, Pisansky TM, Martenson J, Komaki R, Okawara G, Rosenthal SA, Kelsen DP (2002) INT 0123 (Radiation Therapy Oncology Group 94-05) phase III trial of combined-modality therapy for esophageal cancer: high-dose versus standard-dose radiation therapy. J Clin Oncol 20(5): 1167-1174.

Nasu S, Ang KK, Fan Z, Milas L (2001) C225 antiepidermal growth factor receptor antibody enhances tumor radiocurability. Int J Radiat Oncol Biol Phys 51(2): 474-477.

Radiation Therapy Oncology Group (2007) RTOG/EORTC Late Radiation Morbidity Scoring Schema. Available at http://www.rtog.org/ ResearchAssociates/AdverseEventReporting/ RTOGEORTCLateRadiationMorbidityScoringSchema.aspx.

Rees J, Hurt CN, Gollins S, Mukherjee S, Maughan T, Falk SJ, Staffurth J, Ray R, Bashir N, Geh JI, Cunningham D, Roy R, Bridgewater J, Griffiths G, Nixon LS, Blazeby JM, Crosby T (2015) Patient-reported outcomes during and after definitive chemoradiotherapy for oesophageal cancer. Br J Cancer 113(4): 603-610.
Ruhstaller T, Pless M, Dietrich D, Kranzbuehler H, von Moos R, Moosmann P, Montemurro M, Schneider PM, Rauch D, Gautschi O, Mingrone W, Widmer L, Inauen R, Brauchli P, Hess V (2011) Cetuximab in combination with chemoradiotherapy before surgery in patients with resectable, locally advanced esophageal carcinoma: a prospective, multicenter phase IB/II Trial (SAKK 75/06). J Clin Oncol 29(6): 626-631.

Schemper M, Smith TL (1996) A note on quantifying follow-up in studies of failure time. Control Clin Trials 17(4): 343-346.

Shintani S, Li C, Mihara M, Terakado N, Yano J, Nakashiro K, Hamakawa H (2003) Enhancement of tumor radioresponse by combined treatment with gefitinib (Iressa, ZD1839), an epidermal growth factor receptor tyrosine kinase inhibitor, is accompanied by inhibition of DNA damage repair and cell growth in oral cancer. Int $J$ Cancer 107(6): 1030-1037.

Stahl M, Stuschke M, Lehmann N, Meyer HJ, Walz MK, Seeber S, Klump B, Budach W, Teichmann R, Schmitt M, Schmitt G, Franke C, Wilke H (2005) Chemoradiation with and without surgery in patients with locally advanced squamous cell carcinoma of the esophagus. J Clin Oncol 23(10): 2310-2317.

Suntharalingam M, Winter K, Ilson D, Dicker AP, Kachnic LA, Konski AA, Chakravarthy B, Anker CJ, Thakrar H, Horiba N, Kavadi V, Deutsch M, Raben A, Roof M, Videtic G, Pollack J, Safran H, Crane CH (2014) The initial report of local control on RTOG 0436: a phase 3 trial evaluating the addition of cetuximab to paclitaxel, cisplatin, and radiation for patients with esophageal cancer treated without surgery. Int J Radiat Oncol Biol Phys 90(1): S3.

Ubink I, van der Sluis P, Schipper M, Reerink O, Voest E, Borel-Rinkes I, Wijrdeman H, Vleggaar F, Agterof M, Overkleeft E, Siersema P, van Hillegersberg R, Lolkema MP (2014) Adding preoperative radiotherapy plus cetuximab to perioperative chemotherapy for resectable esophageal adenocarcinoma: a single-center prospective phase II trial. Oncologist 19(1): 32-33.

Waddell T, Chau I, Cunningham D, Gonzalez D, Okines AF, Okines C, Wotherspoon A, Saffery C, Middleton G, Wadsley J, Ferry D, Mansoor W, Crosby T, Coxon F, Smith D, Waters J, Iveson T, Falk S, Slater S, Peckitt C, Barbachano Y (2013) Epirubicin, oxaliplatin, and capecitabine with or without panitumumab for patients with previously untreated advanced oesophagogastric cancer (REAL3): a randomised, open-label phase 3 trial. Lancet Oncol 14(6): 481-489.

Wallach JD, Sullivan PG, Trepanowski JF, Steyerberg EW, Ioannidis JP (2016) Sex based subgroup differences in randomized controlled trials: empirical evidence from Cochrane meta-analyses. BMJ 355: i5826.

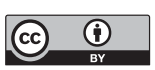

This work is licensed under the Creative Commons Attribution 4.0 International License. To view a copy of this license, visit http://creativecommons.org/licenses/by/4.0/

(C) The Author(s) named above 2017

Supplementary Information accompanies this paper on British Journal of Cancer website (http://www.nature.com/bjc) 